\title{
THE USE OF REMOTE AND TRADITIONAL FACILIATION TO EVALUATE TELESIMULATION TO SUPPORT INTERPROFESSIONAL EDUCATION AND PROCESSING IN HEALTHCARE SIMULATION TRAINING
}

\author{
Coy Collins \\ Marissa Lovett \\ David Biffar Karen Holder \\ Allan Hamilton \\ Arizona Simulation Technology and Education \\ Center \\ University of Arizona \\ 1501 N. Campbell Avenue \\ Tucson, AZ, USA \\ \{coyc,lovett1,dbiffar,allan\}@email.arizona.edu \\ Northern Arizona Health Education Center \\ North Country Healthcare \\ 2920 N. $4^{\text {th }}$ Street \\ Flagstaff, AZ, USA \\ KHolder@nchcaz.org
}

\author{
Mike Holcomb \\ Peter Yonsetto \\ Ronald Weinstein \\ Arizona Telemedicine Program \\ University of Arizona \\ 1501 N. Campbell Avenue \\ Tucson, AZ, USA \\ \{mholcomb,pyonsetto,rweinstein\}@telemedicine.arizona.edu
}

\begin{abstract}
This pilot study appraised traditional versus remote facilitation via telesimulation for an established interprofessional training at two geographically separate sites. Participant feedback was captured via 5point Likert scale surveys. Results demonstrate learners supported the use of remote facilitation: to meet the interprofessional learning objectives, as an adequate replacement for live facilitation and to implement simulation education in low-resource or low-facilitator areas. Improvements were suggested for audio connectivity between participants. In conclusion, the program evaluation suggests that telesimulation, with remote and traditional facilitation, is an effective strategy to provide interprofessional simulation education. Improvements identified are to standardize the set-up of audio/visual technology and tailor participant orientation to encourage meaningful dialogue between sites.
\end{abstract}

Keywords: telesimulation, remote facilitation, interprofessional education, medical simulation 


\section{INTRODUCTION}

Interprofessional education, often implemented through simulation education, has become a primary strategy to improve clinical communication and health outcomes (Khan et al. 2016, Gilbert et al 2010). A prominent program run by the Arizona Simulation Technology and Education Center (ASTEC) for introducing professional entry students to vital elements of interprofessional collaborative practice is the Interprofessional Cardio-Pulmonary Resuscitation (IPCPR) Training. Despite solid support and established longevity of the program, challenges do arise in securing finite resources and expert facilitators in a robust academic setting. Likewise rural educational programs run by the Northern Arizona Area Health Education Center (NAHEC) are inconvenienced by nuanced, but comparable barriers. Telesimulation may provide a new modality to expand interprofessional education to more learners by addressing the key barriers of cost and facilitator deficit within traditional simulation education (Hayden et al. 2018, Sunguya et al. 2014). This program evaluation was designed to appraise the effectiveness of remote facilitation in comparison to traditional facilitation for interprofessional events using telesimulation, and to develop a model for interactive, interprofessional training amongst the geographically separated sites.

\section{MATERIALS AND METHODS}

\subsection{Design}

A small pilot study was carried out using the current IPCPR educational offering (see protocol below). The IPCPR scenario was delivered to two geographically separated sites. Each group in either location had an opportunity to carry out an assigned clinical IPCPR scenario under the guidance of an in-person simulation facilitator and a second opportunity to carry out the scenario with a facilitator in a remote location who interacted through a telesimulation portal. Institutional review board approval was obtained permitting the evaluation of the educational methodology. All participants were voluntary staff and students of the participating institutions.

\subsection{Setting and Population}

The program evaluation was conducted at the ASTEC in Tucson, Arizona, and the NAHEC in Flagstaff, Arizona. It is approximately 260 driven statute miles between the centers. Five first-year medical students participated at ASTEC $(n=5)$, while two second-year osteopathic students and three fourth-year pharmacy students participated at NAHEC $(\mathrm{n}=5)$.

\subsection{Outcome Measures}

The primary outcome of interest is in the quality of facilitation via the remote model. Secondary interest lies within the quality of the telesimulation environment for the learner.

\subsection{Protocol}

Both locations were equipped with a high-fidelity patient simulator, all necessary medical supplies to perform cardiopulmonary resuscitation, and telemedicine visual/audio connectivity via support of the Arizona Telemedicine Program (ATP). Both sites utilized H.323 hardware based video conferencing equipment and MS-Windows based laptops using USB HD cameras and microphones. The Zoom Cloud meeting platform was used to allow both sites to see and hear each other via the internet as well as establish an encrypted, secure connection. NAHEC utilized a Cisco H323 room system with multiple HD cameras, built-in ceiling mics and multiple displays. The ASTEC location utilized MS-Windows based laptops with a Logitech GROUP Videoconferencing System with Expansion Mics and multiple displays. 


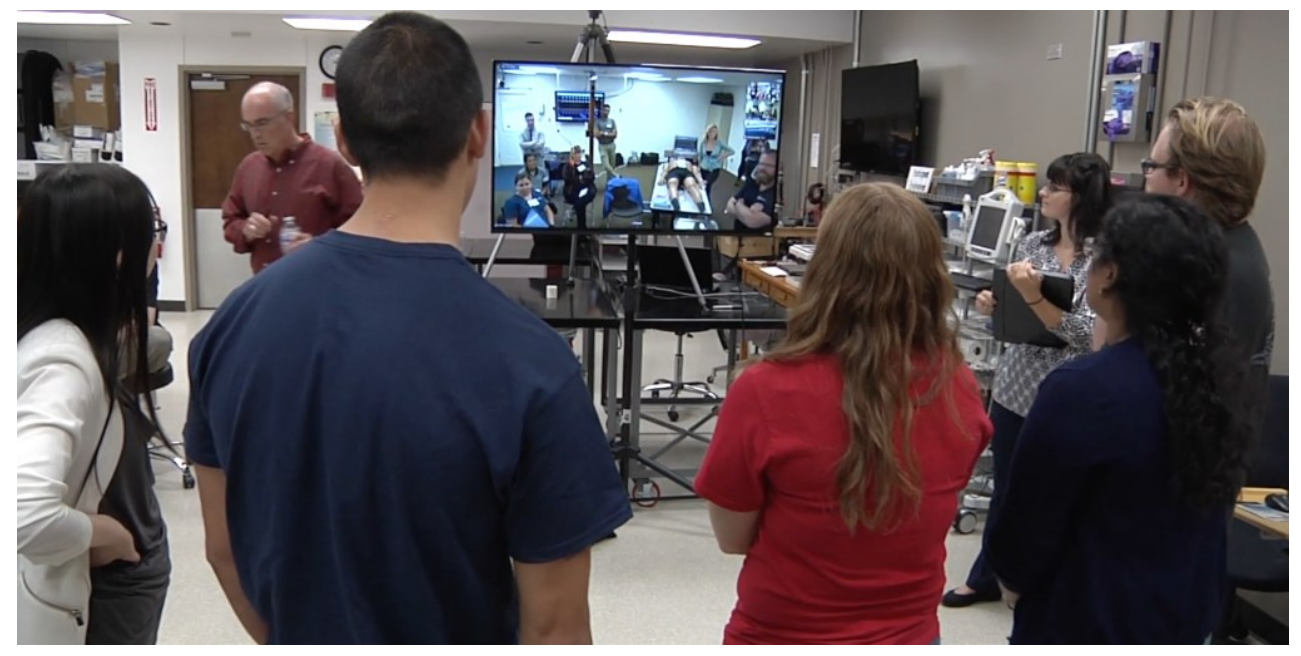

Figure 1: Students at the ASTEC and NAHEC sites participating in a joint debrief.

Participant learners completed two IPCPR scenarios per site, each followed by a post-scenario debriefing session. At each site, one scenario debriefing was led in a traditional fashion by the in-person facilitator and one debriefing was led by the remote facilitator in a cross-over fashion. The two facilitators were experienced in simulation debriefing and have supported numerous IPCPR training sessions in person in the past. Facilitators followed structured debriefs in IPCPR that highlight key concepts surrounding effective team dynamics: clear messages, closed loop communication, clear roles and responsibilities, knowledge sharing, constructive intervention, and mutual respect (Preschner et al. 2014). All participants, regardless of location, were able to contribute meaningful dialogue during the debrief. Overall, four scenarios were completed in the presence of all learners. Participants completed a survey at the end of the training utilizing a 5-point Likert scale soliciting feedback ranging from the from the effectiveness of the training to the quality of the environment and equipment.

\subsection{Analysis}

Microsoft Excel 2016@ was used for all data management and statistics computation.

\section{RESULTS}

10 learners participated in the pilot study, five per each site. $100 \%(\mathrm{n}=10)$ completed surveys within one week post training. Professional discipline entry status was three (3) learners in a pharmacy doctorate tract and seven (7) in a physician provider. Overall experience was comprised of five (5) first-year, two (2) second-year and three (3) fourth year students.

\subsection{Quality of Facilitation}

The pilot study appraised traditional versus remote facilitation via telesimulation for an established interprofessional training at two geographically separate sites. Participant feedback was captured via 5-point qualitative Likert scale surveys with 1 being strongly disagree and 5 being strongly agree, see Table 2 . Results demonstrate learners supported the use of remote facilitation to meet the interprofessional learning objectives $(M=4.9, S D=0.3162)$. While scoring for consideration of remote facilitation as an adequate replacement for live, or traditional, facilitation was lower $(M=4.2, S D=0.6325)$, there is a tendency towards agreeance in its utility. Additionally there is high agreeance in the ability of the remote facilitator to provide a valuable simulation training experience using distance learning technologies $(M=4.9$, $S D=0.3162$ ). 
Table 2: The survey was completed by all learners $(n=10)$ and returned within a week of the simulation training. Learner post-simulation surveys scored on a 5-point Likert scale (1 - Strongly Disagree; 5 Strongly Agree).

\begin{tabular}{ll}
\hline Survey Question: & Mean (SD) \\
\hline I could see who was speaking at the remote site. & $4.5(0.7071)$ \\
$\begin{array}{l}\text { I could follow along as the simulation scenario unfolded } \\
\text { with relative ease. }\end{array}$ & $4.5(0.7071)$ \\
$\begin{array}{l}\text { I could easily identify what each learner was doing } \\
\text { throughout the scenario. }\end{array}$ & $4.3(0.8233)$ \\
$\begin{array}{l}\text { I could hear the discussion between participants clearly. } \\
\text { I could hear the facilitator clearly. }\end{array}$ & $3.9(0.5676)$ \\
$\begin{array}{l}\text { As a remote learner, the objectives for this session were } \\
\text { adequately addressed. }\end{array}$ & $4.9(0.3(0.0000)$ \\
$\begin{array}{l}\text { The facilitator was able to provide a valuable simulation } \\
\text { training experience using distance learning technologies. }\end{array}$ & $4.9(0.3162)$ \\
$\begin{array}{l}\text { The remote facilitation was just as effective as the live } \\
\text { facilitation. }\end{array}$ & $4.2(0.6325)$ \\
$\begin{array}{l}\text { I would recommend using telesimulation (distance } \\
\text { learning using simulation technology) for locations with }\end{array}$ & $4.7(0.6749)$ \\
low resource and low facilitator availability.
\end{tabular}

\subsection{Quality of Telesimulation Environment}

Participants reported that the set-up and operation of telesimulation technology was sufficient for visual connectivity by seeing who was speaking and following along with the scenario $(M=4.5, S D=0.7071)$. The facilitator audio connectivity was highly regarded as clear $(M=5.0, S D=0.0000)$. Improvements were suggested for audio connectivity between participants $(M=3.9, S D=0.5676)$. Overall, there was relatively high agreeance in recommending implementation of telesimulation education in low-resource or lowfacilitator areas $(M=4.7, S D=0.6749)$.

\section{DISCUSSION}

There is an absence of research establishing telesimulation. Most prevalent literature to date on medical telesimulation steers heavily towards psychomotor learning, leaving cognitive and affective domains relatively untouched (Okrainec et al. 2010, Papanagnou 2017). Improving medical skill acquisition and retention typically has rapid impact on outcomes, especially when supporting remote sites that have disparate resources, thereby offering a faster yield (Mikrogianakis et al. 2011, Okrainec 2010). However, a glaring paucity is that the term "telesimulation" does not yet have definitive roots in established professional arenas within the field of healthcare simulation. The Society for Simulation in Healthcare's Healthcare Simulation Dictionary lacks any specific entry for telesimulation (Lopreiato 2016). Adoption of the terminology of "telesimulation" is recommended to refer specifically to the remote conduct of any manner of procedural- or scenario-based simulation training where the learners, facilitator, and/or equipment are separated in physical time and/or space but are connected instead through the means of telecommunications equipment. In this manner, telesimulation will be used in a parallel context to the term telemedicine; in the latter, the remote evaluation, diagnosis, and treatment of a patient is carried out by a healthcare practitioner 
via remote telecommunications technology. Typically, telemedicine is carried out in real time with the patient and healthcare professional in locations remote to each other. However, it should be increasingly recognized that, while such contemporaneous interactions may be beneficial to both patient and practitioner, they may no longer be necessary; i.e., the provider could actually evaluate a patient whose examination and history were obtained and recorded at an earlier time for later evaluation by the clinician.

Technology plays an important role in any telecommunications process. This is particularly critical when it comes to modifications or improvement in visual/audio connectivity is constantly moving forward, and typically parallels an increasing demand by the consumer for a higher quality experience. Our program evaluation utilized technology products all within a 5-year life cycle, yet scored the lowest of all survey questions $(M=3.9, S D=0.5676)$. Emphasis on mobile platforms deserves unique attention, especially based on the penetration of these devices in professional, academic, and personal settings. This is particularly useful when considering that high-value simulation equipment is likely to be unevenly distributed, particularly when comparing simulation training opportunities and facilities amongst urban to and rural settings (Hayden et al. 2018). Developing or leveraging existing so-called smartphone applications to individually support participants in telesimulation groups would appear to be a natural area where individual connectivity could be improved for telesimulation needs, including making it easier to establish audio connection with individual participants. Similarly, using off-the-shelf products such as Zoom ${ }^{\mathrm{TM}}$ or Skype $^{\mathrm{TM}}$ could offer a more less expensive and more accessible platform to telesimulation for low resource areas without tech support.

Improving the familiarity of simulation users with the needs and limitations of telesimulation environments would be helpful to minimizing technological obstacles and enhancing participation of individual learners. Age may play a role too as younger adult learners may find themselves more comfortable with remote presence and interactivity than older learners. In particular, it is vital to be able to evaluate learner objectives and team interactivity in multi-disciplinary, inter-professional simulation training and this requires meaningful dialogue amongst the learners and between learners and their facilitators be impacted as little as possible by the technology itself. This capability in telesimulation was observed within the study participants based on the high agreeance in the ability of the remote facilitator to provide a valuable simulation training experience using distance learning technologies $(M=4.9, S D=0.3162)$. Expanding telesimulation pre-training access for learners to increase familiarity with the technology, providing an orientation video/activity to encourage and guide remote communications, and structuring facilitation to leverage telesimulation are tactics strategies that may be employed to accelerate familiarity and comfort with remote telesimulation training.

The benefits for telesimulation within interprofessional education activities cannot be overstated. The ability to connect learners from different campuses/sites that would alternatively not be able to train together is invaluable. Interprofessional collaboration and education is a rapidly expanding priority within all healthcare disciplines. Telesimulation training also has direct relatability in preparing healthcare providers to function within evolving care modalities that are reliant upon telemedicine for service delivery. This is particularly a growing trend in rural and low resource areas.

Overall, telesimulation training offers a model that is effective in engaging students at multiple locations and expanding access to simulation training. Two major shortages that hamper simulation education are access to (1) trainers and facilitators and (2) equipment. We envision telesimulation as being effective for health care learners who do not have access to facilitators while rental of equipment may also reduce institutional commitments to have specialized simulation equipment on hand at all times.

\subsection{Limitations}

This study was a limited proof of concept exercise and does not allow us to draw any significant conclusions about the effectiveness of such telesimulation in comparison to real-time training with a facilitator on site. The results show that the telesimulation is a possibility without saying anything definitive about its efficacy as a teaching tool. The subject matter used in IPCPR is quite simple in terms of its clinical sophistication 
and focused more on inter-professional communication skills rather than clinical skills per se. Further research into better technology to enhance connectivity will be necessary while working at the same time to ensure that remote facilitation becomes competitive with having facilitators on site.

\section{CONCLUSION}

This pilot study suggests that telesimulation, with remote and traditional facilitation, is an effective strategy to provide interprofessional simulation education. Improvements identified are to standardize the set-up of audio/visual technology and tailor participant orientation to encourage meaningful dialogue between sites. Further research is recommended that focuses on validating the educational platform along with optimizing the technological delivery.

\section{ACKNOWLEDGMENTS}

Arizona Area Health Education Centers (AzAHEC) and North Country Healthcare (NCHC) are pillars in advancing patient care and the educational preparedness of tomorrow's health care team. Their support in this demonstration is greatly appreciated.

\section{REFERENCES}

Gilbert, J., Yan, J., Hoffman, S.. A WHO Report: Framework for Action on Interprofessional Education and Collaborative Practice. Journal of Allied Health, 2010; 39 (Supplement 1): 196-197.

Hayden, E., Khatri, A., Kelly, H., Yager, P., and Salazar, G.. "Mannequin-based Telesimulation: Increasing Access to Simulation-based Education." Academic Emergency Medicine 25, no. 2 (2018): 144-147.

Hayden, M., Khatri, A.., Kelly, H., Yager, P. Salazar, G. Mannequin-based Telesimulation: Increasing Access to Simulation-based Education. Academic Emergency Medicine, 2018; 25:144-147.

Khan, N., Shahnaz, S., Gomathi, K. Currently Available Tools and Teaching Strategies for the Interprofessional Education of Students in Health Professions: Literature review. Sultan Qaboos University Medical Journal. 2016;16(3):e277-e285.

Lopreiato, J. Healthcare Simulation Dictionary. Rockville, MD: Agency for Healthcare Research and Quality; October 2016. AHRQ Publication No. 16(17)-0043.

Mikrogianakis, A., Kam, A., Silver, S., Bakanisi, B., Henao, O., Okrainec, A., and Azzie, G. "Telesimulation: an innovative and effective tool for teaching novel intraosseous insertion techniques in developing countries." Academic Emergency Medicine 18, no. 4 (2011): 420-427.

Okrainec, A., Oscar, H., and Georges, A. "Telesimulation: an effective method for teaching the fundamentals of laparoscopic surgery in resource-restricted countries." Surgical endoscopy 24, no. 2 (2010): 417-422.

Papanagnou, D. "Telesimulation: A Paradigm Shift for Simulation Education." AEM Education and Training 1, no. 2 (2017): 137-139.

Preschner, H., Biffar, D., Tomasa, L., Theodorou, A., Berg, M., Grisham, L., Don, L., Hamilton, A. 2014. Breaking Barriers in Health Care Education: The Impact of Interprofessional CPR (IPCPR) Training. Available http://astec.arizona.edu/sites/astec.arizona.edu/files/images/IMSH\%202014\%20IP\%20CPR\%20Poste r.pdf. Accessed Jan. 7, 2019.

Sunguya, B., Hinthong, W., Jimba, M., Yasuoka, J.. Interprofessional Education for Whom? - Challenges and Lessons Learned from Its Implementation in Developed Countries and Their Application to Developing Countries: A Systematic Review. Zunt JR, ed. PLoS ONE. 2014;9(5):e96724. 


\section{AUTHOR BIOGRAPHIES}

COY COLLINS is an Medical Simulation Specialist in the Arizona Simulation Technology and Education Center (ASTEC) at the University of Arizona. He holds an MSN in Family Nurse Practitioner studies from Northern Arizona University. His research interests lie in interprofessional education and simulation based learning, especially in mobile integrated healthcare (MIH) settings. His email address is ccollins@surgery.arizona.edu.

MARISSA LOVETT is a Medical Student at the University of Arizona College of Medicine - Tucson and a Research Specialist at the Arizona Simulation Technology and Education Center (ASTEC). She holds an MS in Physiology from the University of Arizona. Her research interests include optimization of telesimulation training, evaluation of optical medical technology, and design and testing of augmented reality applications to enhance medical education. Her email address is Lovett1@email.arizona.edu.

DAVID BIFFAR is the Director of Operations for the Arizona Simulation Technology and Education Center (ASTEC) and the Center for Advanced Technologies in Healthcare (CATH). He is a Certified Healthcare Simulation Operations Specialist (CHSOS) and an Accreditation Reviewer for the Society for Simulation in Healthcare. His research interests include human factors and usability testing in healthcare environments, interprofessional education modalities, and virtual reality exposure therapy for generalized anxiety disorders. His email address is dbiffar@surgery.arizona.edu.

KAREN HOLDER is a Family Nurse Practitioner, Certified Nurse Midwife, interdisciplinary preceptor for graduate students in health professions, and is the interprofessional education coordinator for North Country Healthcare. She plans and coordinators IPE activities and events in collaboration with several Universities throughout Arizona, and works with students face to face and via telehealth platforms. Her email address is KHolder@nchcaz.org.

MICHAEL HOLCOMB is the Associate Director of Information Technology for the Arizona Telemedicine Program at the University of Arizona. His interests include leveraging high-quality video, voice and data communications solutions to enable clinical telemedicine and telehealth services, and health sciences research, education, and administration applications. He holds a BS in Management Information Systems from the University of Arizona. His email address is mholcomb@telemedicine.arizona.edu.

PETER YONSETTO received a BS from the University of Arizona and is currently the Video Conferencing Administrator with the Arizona Telemedicine Program. His interests include the multidisciplinary approach and dynamics involved in simulation educational events incorporating virtual learning and related technologies. His email address is pyonsetto@telemedicine.arizona.edu.

RONALD WEINSTEIN, MD is a Professor of Pathology and the Director of the Arizona Telemedicine Program. His current research interests are evaluating the necessity of present, on-site simulation facilitators versus tele-esimulation with remote simulation facilitators. His email address is rweinstein@telemedicine.arizona.edu.

ALLAN HAMILTON, MD, FACS is the Executive Director for the Arizona Simulation Technology and Education Center (ASTEC) and a Professor of Neurosurgery at the University of Arizona College of Medicine - Tucson. His current research interests include interprofessional education modalities and virtual patient simulation. His email address is allan@surgery.arizona.edu. 